\title{
Novel Method for Evaluating Spatial Resolution of Magnetic Resonance Images
}

\section{tomokazu takeuchi ( $\nabla$ tm.takeuchi67@gmail.com )}

Gunma Prefectural College of Health Sciences https://orcid.org/0000-0002-0623-9974

\section{Norio Hayashi}

Gunma Prefectural College of Health Sciences

\section{Yuta Asai}

Nippon Medical School Hospital

\section{Yuka Kayaoka}

Osaka City University Hospital

Kiichi Yoshida

Nippon Medical School Hospital

\section{Research Article}

Keywords: MRI, Image quality assessment, spatial resolution, MTF, ladder method

Posted Date: August 23rd, 2021

DOI: https://doi.org/10.21203/rs.3.rs-823953/v1

License: (c) (i) This work is licensed under a Creative Commons Attribution 4.0 International License. Read Full License

Version of Record: A version of this preprint was published at Physical and Engineering Sciences in Medicine on March 1st, 2022. See the published version at https://doi.org/10.1007/s13246-022-01114-6. 


\section{Novel method for evaluating spatial resolution 5 of magnetic resonance images}

6

7

8

9

10
Tomokazu Takeuchi 1)

Norio Hayashi 1)

Yuta Asai 2)

Yuka Kayaoka 3)

Kiichi Yoshida 2)

1) Graduate School, Gunma Prefectural College of Health Sciences

2) Department of radiology, Nippon Medical School Hospital

3) MedCity21, Division of Premier Preventive Medicine, Osaka City University

Hospital

Corresponding Author: Tomokazu Takeuchi Graduate School, Gunma Prefectural College of Health Sciences 1-22-1, Mitumata-machi, Maebashi, Gunma, JAPAN TEL: +8190 8187-9988 E-mail address: tm.takeuchi67@gmail.com

\section{Acknowledgements}

I am grateful to Dr. A. Ogura for giving me constructive comments and warm

encouragement. I also thank Dr. T. Miyati for clear advice and comments. 
30 Abstract

$31 \quad$ Purpose

32 Recently, several methods for evaluating the spatial resolution of magnetic

33 resonance imaging (MRI) have been reported. However, these methods are not simple

34 and can only be used for specific devices. The International Electrotechnical

35 Commission (IEC) 62464-1 recommends a method that uses a periodic array pattern to

36 evaluate the spatial resolution of an MRI device. In this study, we develop a new

37 method (the ladder method) and evaluate its measurement accuracy by adapting the IEC

38 method to evaluate the spatial resolution.

39 Methods

40 First, the adaptation of the IEC method is analyzed by simulating the ladder

41 method using a phantom with a periodic pattern, which is constructed using acrylic

42 plates and a nickel sulfate aqueous solution. Subsequently, the ladder method is

43 evaluated in terms of spatial resolution by dividing the standard deviation (SD) by the

44 average signal in the region of interest (ROI) on the ladder phantom image. To evaluate

45 the precision of the ladder method, it is compared with the modulation transfer function

46 (MTF) using a magnitude image with the partial volume effect of the edge image.

$47 \quad$ Results 
The simulation result shows that the evaluation of the spatial resolution using the ladder method is viable, in which a coefficient of correlation of 0.90 or higher is obtained for all evaluations using the ladder and MTF methods.

\section{Conclusions}

53 ROI on the target image. Therefore, the ladder method is a promising method as a

54 substitute for the MTF.

56 Keywords: MRI, Image quality assessment, spatial resolution, MTF, ladder method

58 Declarations

59 Funding: No finding was received.

60 Conflicts of interest/Competing interests: The author declared that they have no conflict

61 of interest.

62 Availability of data and material: Not applicable.

63 Code availability: Not applicable.

64 Author's contributions: NH made the substantial contributions to the conception. YA,

65 YK and KY interpreted the phantom data. 
66 Ethics approval: Not applicable.

67 Consent to participate: Not applicable.

68 Consent for publication: Not applicable.

69

70

71

72

73

74

\section{Introduction}

Recently, imaging methods for magnetic resonance imaging (MRI) that are

optimized for clinical use have been developed. In these processes, image quality assessment is often performed, where the signal-to-noise ratio, contrast properties, and spatial resolution are considered. The modulation transfer function (MTF) is used the most owing to its definition properties, which are quantifiable and highly precise [1-6]. However, the magnitude signal obtained from MRI using the MTF cannot satisfy systems whose output scales linearly with input (linearity). This problem renders it difficult to determine the MTF of MRI [7-10]. In some studies, signals obtained prior to the absolute value arithmetic operation were used to calculate the correct MTF; however, 
84 these methods are not simple [10]. that uses the magnitude image to assess the spatial resolution of MRI [11]. This method uses periodic pattern images to set the region of interest (ROI) on the image.

Subsequently, $\mathrm{m}(\mathrm{v})$ at the spatial resolution $\mathrm{v}[$ cycles $/ \mathrm{mm}]$ is calculated using the pixel signal mean (S) and SD inside the ROI, as follows:

$$
\mathrm{m}(\mathrm{v})=\mathrm{SD} / \mathrm{S}
$$
single spatial frequency because it is used to assess the periodic resolution of a specific

94 MRI device. Hence, it is unsuitable for assessing the resolution of different imaging sequences. In this study, we developed a method to evaluate the spatial resolution of various MRI images using a new application of IEC 62464-1. We named this method the validity of the ladder method and establish it as a viable method for evaluating the spatial resolution of MRI. 
2. Material and methods

\subsection{Analysis of simulation using ladder method}

Our proposed method for evaluating spatial resolution is a modification of the IEC

62464-1 method. The method recommended by the IEC includes determining the image

quality to ascertain and maintain the performance evaluation of medical devices. The

phantom used for this method contains both high and low magnetic resonance (MR)

signal layers to obtain a periodic pattern image. An elliptical ROI is set on the periodic pattern inside the most lateral plate. The S and SD of the ROI are calculated. The $\mathrm{m}(\mathrm{v})$ at spatial frequency $\mathrm{v}$ [cycles/mm] is defined as shown in Equation (1).

The spatial frequency $v$ is calculated using the thickness (L) via Equation (2).

$$
\mathrm{v}=1 / \mathrm{L}
$$

It is noteworthy that IEC 62464-1 restricted the phantom plate thickness and

117 Figure 1 . The IEC recommends a da/L ratio of between 0.61 and 0.70 . 
121 simulation, the suitable da/L will be obtained. In the simulation, the signal was assumed

122 to be that of the pixel arrayed in one dimension, and we accounted for the ideal and

123 blurry images. Simulations based on different da/L values and plate thicknesses were

124 performed with a high signal of 100 and a low signal of 0 . Figure 2 shows the signal

125 array for a plate thickness of $2 \mathrm{~mm}$ and different da/L values used in the simulation.

126 When the one-pixel signal was set as $\mathrm{x}_{\mathrm{i}}$, the adjoining pixel signals was represented as

$127 \mathrm{x}_{\mathrm{i}+1}, \mathrm{x}_{\mathrm{i}+2}, \ldots \mathrm{x}_{\mathrm{i}+(\mathrm{n}-1)}$. Using these pixel signals, the signal mean value $\bar{x}$ was calculated

128 using Equation (3).

$$
\bar{x}=\frac{1}{n} \sum_{i=1}^{n} x_{i}
$$

The SD, $\sigma_{x}$, was calculated using Equation (4), as follows:

$$
\sigma_{x}=\sqrt{\frac{\sum_{i=1}^{n}\left(x_{i}-\bar{x}\right)^{2}}{n}}
$$

An blurry image was obtained by convoluting the convolution filter to the ideal

signal. The convolution filter was a three-term filter whose weight ratio was 1:2:1.

The results of these simulations showed that by changing the spatial frequency (v), $\mathrm{m}(\mathrm{v})$ indicated the most significant change at $\mathrm{da} / \mathrm{L}=0.50$. This implies that $\mathrm{da} / \mathrm{L}=0.50$ can present a detailed resolution difference. Subsequently, another simulation was performed in which the phantom da $/ \mathrm{L}=0.50$, and the plate thickness was set to $1.0,1.5$, 
arrangement, sharp and blurry images were assumed. A blurry image was assumed by convoluting the convolution filter. The weight ratios of the convolution filter were changed to four stages, i.e., $1: 1.5: 1,1: 2: 1,1: 3: 1$, and $1: 4: 1$, where the $1: 4: 1$ ratio presented a sharp image, and the 1:1.5:1 presented the most blurred image (Figure 3).

\subsection{Ladder method}

The phantoms containing periodic patterns, as shown in Figure 1, were used to verify the ladder method. The phantoms comprised 10 parallel acrylic plates. The plate thickness $d$ and the distance between plates da were set to the same value. To achieve parallel acrylic plates and to maintain a certain distance between the plates, a $15 \mathrm{~mm}$ wide acrylic plate was sandwiched on both sides of the plates and then adhered using an acrylic resin (Figure 1). Similar to the IEC method, the plate width (a) was set as twice the slice thickness, and the plate length (b) was set ten times the length L, as shown in Figure 1. Six different phantoms with plate thicknesses of 1, 1.5, 2, 2.5, 3, and $4 \mathrm{~mm}$ were fixed in a nickel sulfate aqueous solution $\left(1.25 \mathrm{~g} \mathrm{NiSO}_{4} \cdot 6 \mathrm{H}_{2} \mathrm{O}+1000 \mathrm{~g} \mathrm{H}_{2} \mathrm{O}\right)$. The details of the custom-developed phantom are shown in Table 1, and a custom-developed phantom with a plate thickness of $2 \mathrm{~mm}$ is shown in Figure 4a. Each phantom was constructed using sections that were cut from the same acrylic plates. 
157 Hence, the accuracy of the phantom construction was high, as the different phantoms

158 did not yield significantly different results. The angle between the phantom's major axis

159 (x-axis) and the magnetic bore was $13^{\circ}$ in the initial setup. The frequency-encoding

160 direction was matched to the $\mathrm{x}$-direction of the phantom, as shown in Figure $4 \mathrm{~b}$. For the

161 imaging, the slice was set on the $\mathrm{x}-\mathrm{z}$ plane, as shown in Figure 1, to obtain the image

162 shown in Figure 4c. The scanning parameters are shown in Section 2.4 below.

163 For the analysis, the ROI was set on the periodic images. The size of the ROI was

164 set to the maximum; however, it did not exceed the circumference of the plates of the

165 phantom and encompassed less than $90 \%$ of the phantom length. The S and SD of all

166 pixels in the ROI were determined. The ladder method evaluates the spatial resolution

167 by dividing the SD by the $\mathrm{S}$ in the ROI on the ladder phantom image using Equation (1).

168 The S and SD in the ROI were measured using ImageJ (National Institutes of Health,

169 Bethesda, MD), whereas Excel (Microsoft Corporation, Washington, USA) was used to

170 calculate $\mathrm{m}(\mathrm{v})$.

The ladder method uses the same periodic pattern phantom and involves an

analysis based on IEC 62464-1. However, the da/L ratio of the phantom was 0.50 , and characteristics. 


\subsection{Calculation of MTF}

The MTF was calculated for a comparison with the ladder method. To obtain the

178

179

180

181

182

183

184

185

186 measured $40 \mathrm{~mm}$ along the $\mathrm{x}$-axis, and its length was $150 \mathrm{~mm}$ (Figure $5 \mathrm{e}$ ). To obtain the correct MTF, we used the partial volume effect [10].

An acrylic plate with a thickness of $20 \mathrm{~mm}$ was fixed in a nickel sulfate aqueous solution to evaluate the MTF (Figure 5a). Figure 5b shows the details of the edge phantom and the location of the slice used for imaging, whereas Figure $5 \mathrm{c}$ shows a photograph of the edge phantom. The slice was set in the middle of the acrylic plate and nickel sulfate aqueous solution on the y-axis to offset the edge spread function (ESF) baseline and eliminate all negative lobes. This setting based on the partial volume effect yielded MTF linearity [10]. For imaging, the phantom edge, which was located in the middle of the $\mathrm{z}$-axis, was set parallel to the magnetic bore (Figure 5b). The $\mathrm{x}$ - and frequency-encoding directions of the images were matched (Figure (5b)). Images of the edges were obtained using the scanning parameters, as shown in Section 2.4, and Figure $5 \mathrm{~d}$ shows the obtained MR image. For the analysis, the square of the ROI was set on the edge of the image, and the ESF was obtained from the profile inside the ROI. The ROI MTF, we first differentiated the ESF using Equation (5), i.e., we performed 
193 finite-element differentiation (FED), and obtained a line spread function (LSF) [10].

$$
\operatorname{LSF}\left(X_{j}\right)=\operatorname{ESF}\left(X_{j}\right)-\operatorname{ESF}\left(X_{j-1}\right),
$$

195 where $\mathrm{X}$ is the spatial location, and $\mathrm{j}$ is the number of samples. Finally, a Fourier was calculated [12].

$$
\alpha(f)=\frac{1}{\operatorname{sinc}\left(\frac{\pi f}{2 f c}\right)}, f c=\frac{f s}{2},
$$

where $\operatorname{MTF}(\mathrm{f})=$ true $\mathrm{MTF}, \operatorname{MTFe}(\mathrm{f})=$ experimental $\mathrm{MTF}$, fc is the Nyquist frequency, calculate the edge profile. The MTF was calculated using Excel.

\subsection{Imaging parameter}

All scans were performed using the abovementioned phantom with a loading

Systems, Best, The Netherlands). Data were collected using single slices via a spin echo 
211 on conventional magnitude imaging, with Repetition Time (TR) $=1000 \mathrm{~ms}$, Echo Time

$212($ TE $)=50 \mathrm{~ms}$, slice thickness $=5 \mathrm{~mm}$, and field of view $=256 \mathrm{~mm} \times 256 \mathrm{~mm}$. IEC

213 62464-1 recommends an Signal to Noise Ratio (SNR) greater than 50. All images used

214 for the ladder method had SNRs of 50 or higher when the number of excitations (NEX)

215 was 1 . Hence, the NEX was set to 1 . The matrix size was varied to obtain images with

216 different resolutions. The 10 different matrix sizes used were 112, 128, 160, 192, 256,

$217288,352,384,448$ and 512 (Table 2). Using Equation (2), the spatial resolution was

218 evaluated at spatial frequencies of $0.125,0.167,0.200,0.250,0.333$, and 0.500

219 cycles/mm using the six different phantoms. The results are presented in Table 3.

220 The imaging parameters for the edge phantom were similar to those used in the

221 ladder method. However, the NEX was set to 15 to reduce noise.

222

223

\subsection{Correlation between ladder method and MTF}

224

The $\mathrm{m}(\mathrm{v})$ and MTF were calculated for each of the 10 different resolutions. To

compare between the $\mathrm{m}(\mathrm{v})$ and MTF, a cutoff frequency, which corresponds to a

specific value, was used [13].

Using the calculated $\mathrm{m}(\mathrm{v})$ and MTF, the cutoff frequencies at $\mathrm{m}(\mathrm{v})$ and MTF 
correlation coefficient between $\mathrm{m}(\mathrm{v})$ and MTF cutoff frequency was compared for each value. The correlation coefficient was calculated using Excel, and then the correlation between $\mathrm{m}(\mathrm{v})$ and MTF cutoff frequency was evaluated.

\section{Results}

\subsection{Simulation using ladder phantom}

The $\mathrm{m}(\mathrm{v})$ values measured at three different ratios of $\mathrm{da} / \mathrm{L}(0.50,0.67$, and 0.80$)$ and three plate thicknesses d (1.0, 2.0, and $3.0 \mathrm{~mm})$ are shown in Figure 6a. The results indicate that the $\mathrm{m}(\mathrm{v})$ of the phantom with $\mathrm{da} / \mathrm{L}=0.50$ varied the most with different plate thicknesses. Therefore, the phantom with $\mathrm{da} / \mathrm{L}=0.50$ was used in the ladder method.

Furthermore, the simulation results obtained by assuming a completely sharp image based on $\mathrm{m}(\mathrm{v})$ at different spatial resolutions when $\mathrm{da} / \mathrm{L}=0.5$ are shown in Figure $6 \mathrm{~b}$. The evaluated results of $\mathrm{m}(\mathrm{v})$ did not vary from the maximum value of 1 at different spatial frequencies owing to the changes in the plate thickness. Figure $6 \mathrm{c}$ shows the results of the blurry pattern using four different convolution filters. These results show that $\mathrm{m}(\mathrm{v})$ decreased at high spatial frequencies and increased at high resolutions. 


\subsection{Ladder method}

The $\mathrm{m}(\mathrm{v})$ values obtained using the ladder method for each plate thickness in the as the spatial frequency increased. The $\mathrm{m}(\mathrm{v})$ was low in the low-resolution images, similarly for the small matrix sizes.

\subsection{MTF} the evaluated $\mathrm{m}(\mathrm{v})$, the MTF curve is shown until a spatial frequency of $0.6 \mathrm{cycles} / \mathrm{mm}$.

\subsection{Correlation between ladder method and MTF}

Figures $9 a-9 c$ show the relationship between $m(v)$ and the MTF cutoff frequency at $\mathrm{m}(\mathrm{v})$ and MTF values of $0.3,0.5$, and 0.8 , respectively, in the 10 different resolution images. The regression lines and correlation coefficients (r) were calculated and are 
265 for 0.5 , and 0.797 for 0.8 . These values indicate a strong correlation between $\mathrm{m}(\mathrm{v})$ and

266 the MTF.

Table 4 shows the correlation coefficient between $\mathrm{m}(\mathrm{v})$ and the MTF cutoff

268 frequency at $0.3,0.4,0.5,0.6,0.7$, and 0.8 . At all spatial frequencies, the correlation

269 coefficient exceeded 0.90 , indicating strong correlation.

270

271

272

4. Discussion

273

Spatial resolution has been evaluated extensively using various methods [1,2].

274 However, these methods are complex. Therefore, we propose a simpler "ladder method"

275 to evaluate the spatial resolution of MRI. The ladder method evaluates the spatial

276 resolution by dividing the SD by the average signal in the ROI on the phantom image of

277 the ladder. We compared the ladder method and MTF to validate the ladder method.

278

The simulation results show that the $\mathrm{m}(\mathrm{v})$ of the phantom with $\mathrm{da} / \mathrm{L}=0.50$ varied

the most with different plate thicknesses. The phantom with da/ $\mathrm{L}=0.50$ can be assumed

to be rectangular waves with a duty ratio of $1: 1$. This rectangular wave contained the 
283 repetition frequency and the frequency of odd and even harmonics. The ladder method

284 evaluates the spatial resolution at a single spatial frequency based on a single phantom;

285 hence, using a phantom that contains many frequencies is not suitable for evaluating the

286 spatial frequency of a pin point. As such, the $\mathrm{m}(\mathrm{v})$ value of the phantom with $\mathrm{da} / \mathrm{L}=$

2870.50 varied the most with different plate thicknesses. Therefore, it was clear that the

288 phantom with $\mathrm{da} / \mathrm{L}=0.50$ was the most suitable for evaluating the spatial resolution in

289 different imaging sequences. With regard to the ladder phantom characteristics, the IEC

290 suggests a da/L of $0.61-0.70$, based on the relationship with the MTF. However, the

291 simulation results indicate that $\mathrm{da} / \mathrm{L}=0.50$ is applicable and renders it easy to

292 understand the phantom characteristics, i.e., the phantom periodicity is $1: 1$.

293 The $\mathrm{m}(\mathrm{v})$ value was obtained by dividing the SD by $\mathrm{S}$ in the ROI. The edge profile

294 of the phantom plate became blurred, and gradation occurred in the low-resolution

295 images. This gradation decreased the SD and hence $\mathrm{m}(\mathrm{v})$. For images of the same

296 resolution with thin plate thicknesses, the phantom cycles become finer and increased

297 the gradation. Hence, m(v) decreased. For phantoms with thin plate thicknesses, the

298 ladder method can evaluate high-frequency spatial resolutions based on Equation (2).

Theoretically, the maximum value of $m(v)=1$ can be obtained using Equation (8) 
results for the ideal case.

$$
\mathrm{m}(\mathrm{v})=\sqrt{\frac{(L-d)}{d}}
$$

Therefore, $\mathrm{m}(\mathrm{v})$ might be correlated to the MTF at low spatial frequencies.

Based on the comparison, the correlation coefficient between $\mathrm{m}(\mathrm{v})$ and the MTF cutoff frequency exceeded 0.90 for all values of m(v) and MTF, which indicated a strong correlation. Therefore, the ladder method is a useful yet easy method for evaluating spatial resolution.

In this study, we used plate thicknesses of $1,1.5,2,2.5,3$, and $4 \mathrm{~mm}$. However, it is unclear whether these configurations are sufficient for the spacing requirements. Hence, different plate thicknesses should be used to evaluate the spatial resolution in future studies.

Whereas several different resolution images were evaluated in this study, images with different contrasts or distortions were not analyzed. Some issues may emerge when using different imaging sequences; therefore, more investigations are required to expand the applicability of this method.

\section{Conclusion}



that can be a substitute for the MTF.

324

325

\section{Compliance with Ethical Standards}

327 Conflict of Interest: The authors declare that they have no conflict of interest.

328 Statement of Human Rights: This article does not contain any studies with human

329 participants performed.

330 Statements of Animal Rights: This article does not contain any studies with animals

331 performed.

332

333

References

1. Delakis L, Xantthis C, Kitney Rl. Assessment of the limiting spatial resolution of an MRI scanner by direct analysis of the edge spread function. Med Phys. 

2009;36:1637-1642.

338

2. Borri M, Scurr ED, Richardson C, Usher M, Leach MO, Schmidt MA. A novel approach to evaluate spatial resolution of MRI clinical images for optimization and standardization of breast screening protocols. Med Phys. 2016;43:6354-6363.

3. Lim WT, Jung HR. Evaluation on availability of MTF measurement using the ACR phantom in MRI. Indian Journal of Science and Technology. 2016;9:1-5.

4. Mohapatra SM, Turley JD, Prince JR, Blechinger JC, Wilson DA. Transfer function measurement and analysis for a magnetic resonance imager. Med Phys. $1991 ; 18: 1141-1144$.

5. Steckner MC, Drost DJ, Plato FS. A cosine modulation artifact in modulation transfer function computations caused by the misregistration of line spread profiles. Med Phys. 1993;20:469-473.

6. Cunningham IA, Reid BK. Signal and noise in modulation transfer function determinations using the slit, wire, and edge techniques. Med Phys. 1992;19:1037-1044.

7. Miyati T, Fujita H, Kasuga T, et al. Measurements of MTF and SNR(f) using a subtraction method in MRI. Phys Med Biol. 2002;47:2961-2972.

8. Steckner MC. Magnitude reconstruction distorts Gibb's phenomenon in magnetic 
resonance imaging. Proceedings of the Society of Magnetic Resonance Imaging (SMRM), 9th Annual Meeting (SNRM, New York, 1990), p. 565.

9. Steckner MC, Drost DJ, Prato FS. Comments and reply:' “Transfer function measurements and analysis for a magnetic resonance imager"' (Mohapatra et al. 1991). Med. Phys. 1992;19;511-512.

10. Steckner MC, Drost DJ, Prato FS. Computing the modulation transfer function of a magnetic resonance imager. Med Phys. 1994;21:483-489.

11. IEC 62464-1, Magnetic resonance equipment for medical imaging - Part 1.

12. Cunningham IA and Fenster A. A method for modulation transfer function determination from edge profile with correction for finite-element differentiation. Med Phys. 1987;14;533-537.

13. Miyoung Kwon, Gordon Legge. Spatial-frequency cutoff requirements for pattern recognition in central and peripheral vision. Vision Research. 2011;51;1995-2007. 
Figures

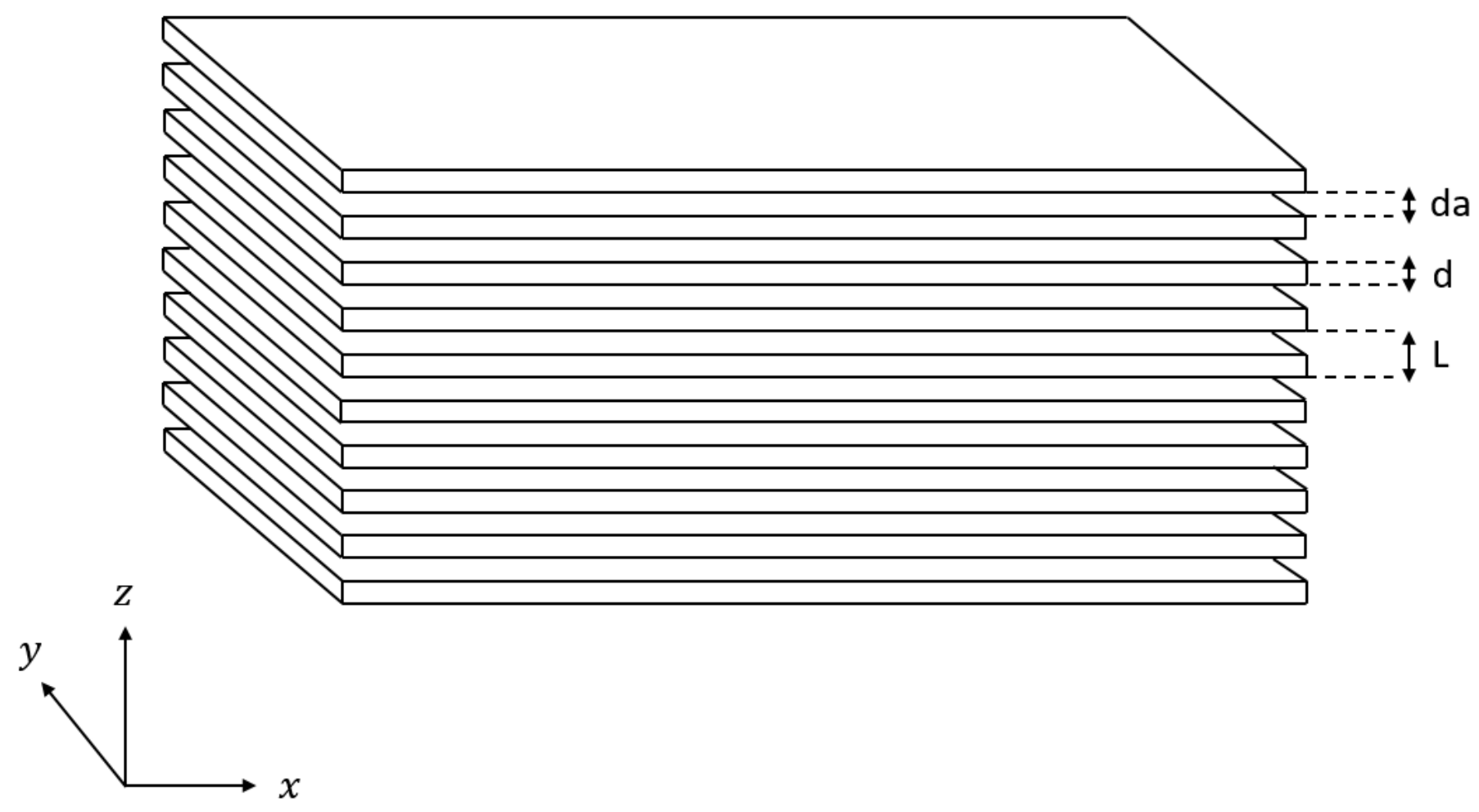

Figure 1

Diagram of phantom used for ladder method 

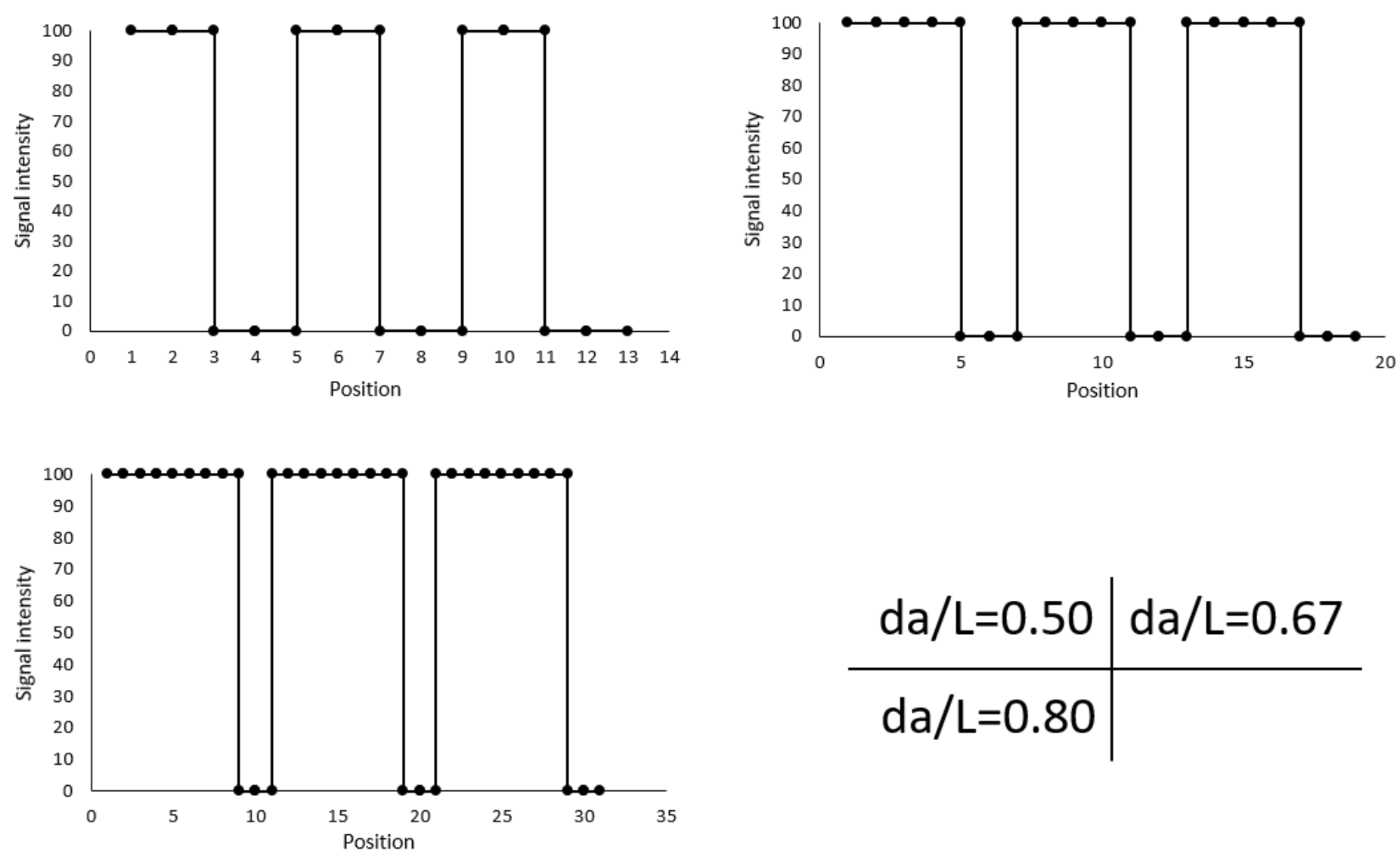

Figure 2

Signal array at $2 \mathrm{~mm}$ plate thickness in different da/L. 


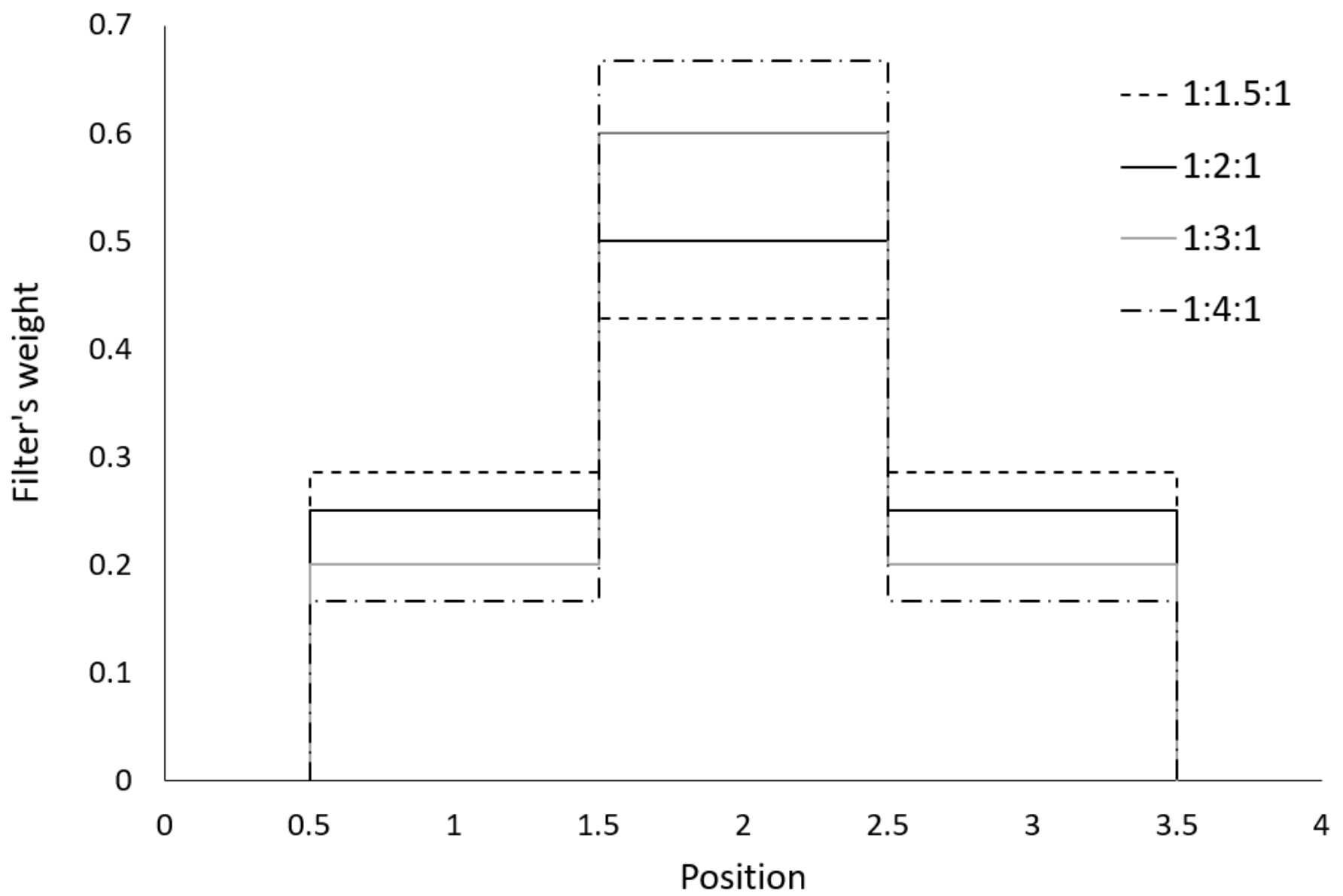

Figure 3

Convolution filter of different weights.
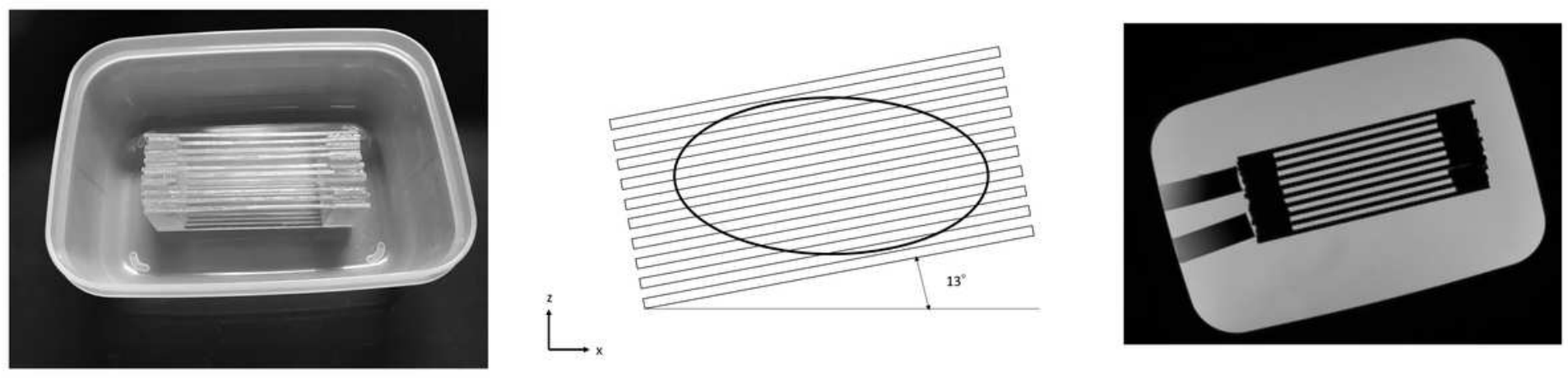

\section{Figure 4}

a. Periodic pattern phantom with plate thickness of $2 \mathrm{~mm}$. b. Placement of ROI on phantom image for ladder method. c. Magnetic resonance image of periodic pattern phantom with plate thickness of $2 \mathrm{~mm}$. 

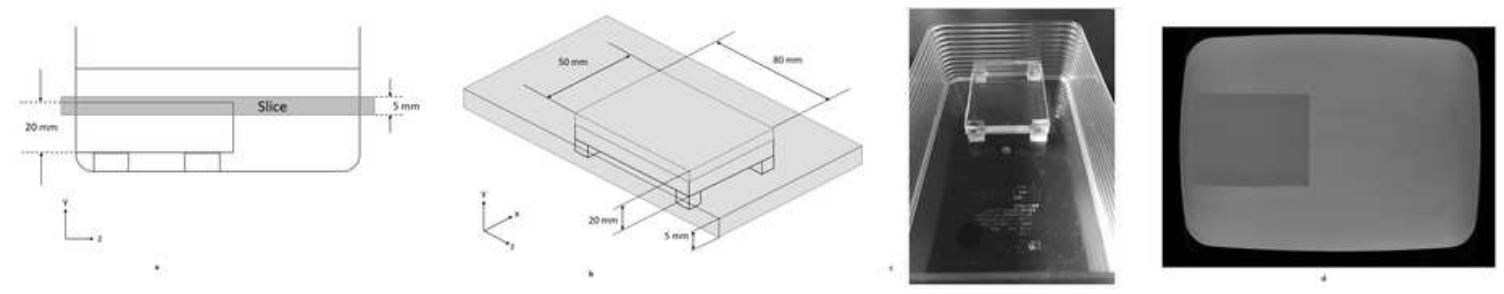

\section{Figure 5}

a. Diagram of edge phantom in y-z plane for calculating MTF. b. Detailed diagram of edge phantom shown in three dimensions. c. Custom-developed edge phantom. d. Magnetic resonance image of edge phantom with matrix size of 512. e. Placement of ROI on edge phantom image to calculate MTF.
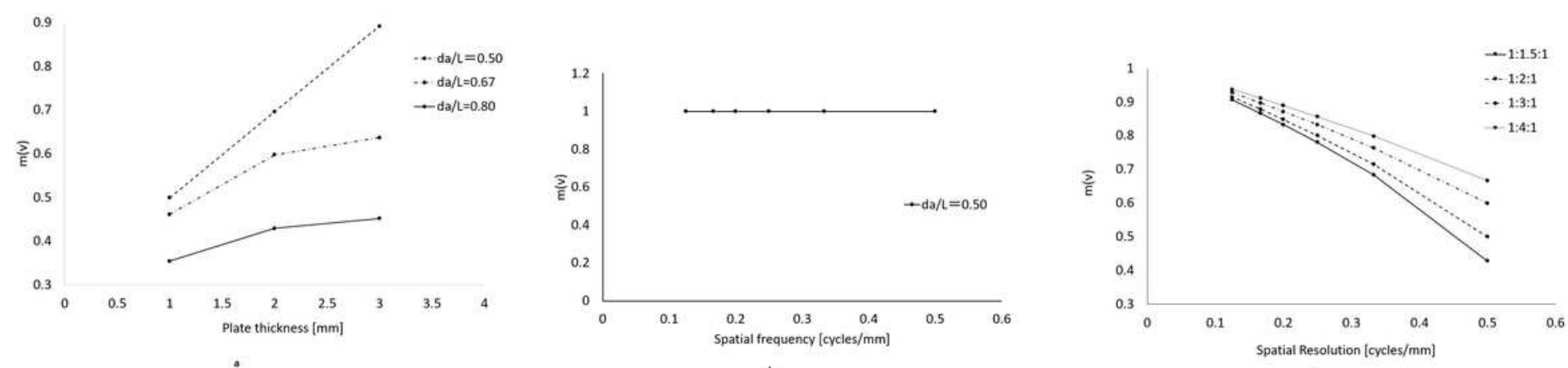

\section{Figure 6}

a. Relations between plate thickness and evaluated value of $\mathrm{m}(\mathrm{v})$ in simulation for different $\mathrm{da} / \mathrm{L}$ values. Evaluated value of $\mathrm{m}(\mathrm{v})$ at $\mathrm{da} / \mathrm{L}=0.50$ shows the most significant change by changing plate thickness. b. Relationship between spatial frequency and evaluated $m(v)$ in simulation of sharp edge; $m(v)$ did not depend on spatial frequency. c. Relationship between spatial frequency and evaluated $m(v)$ in simulation of blurred edge, and Resolution was changed in four variations; $\mathrm{m}(\mathrm{v})$ at high and low spatial frequencies indicated low values. 


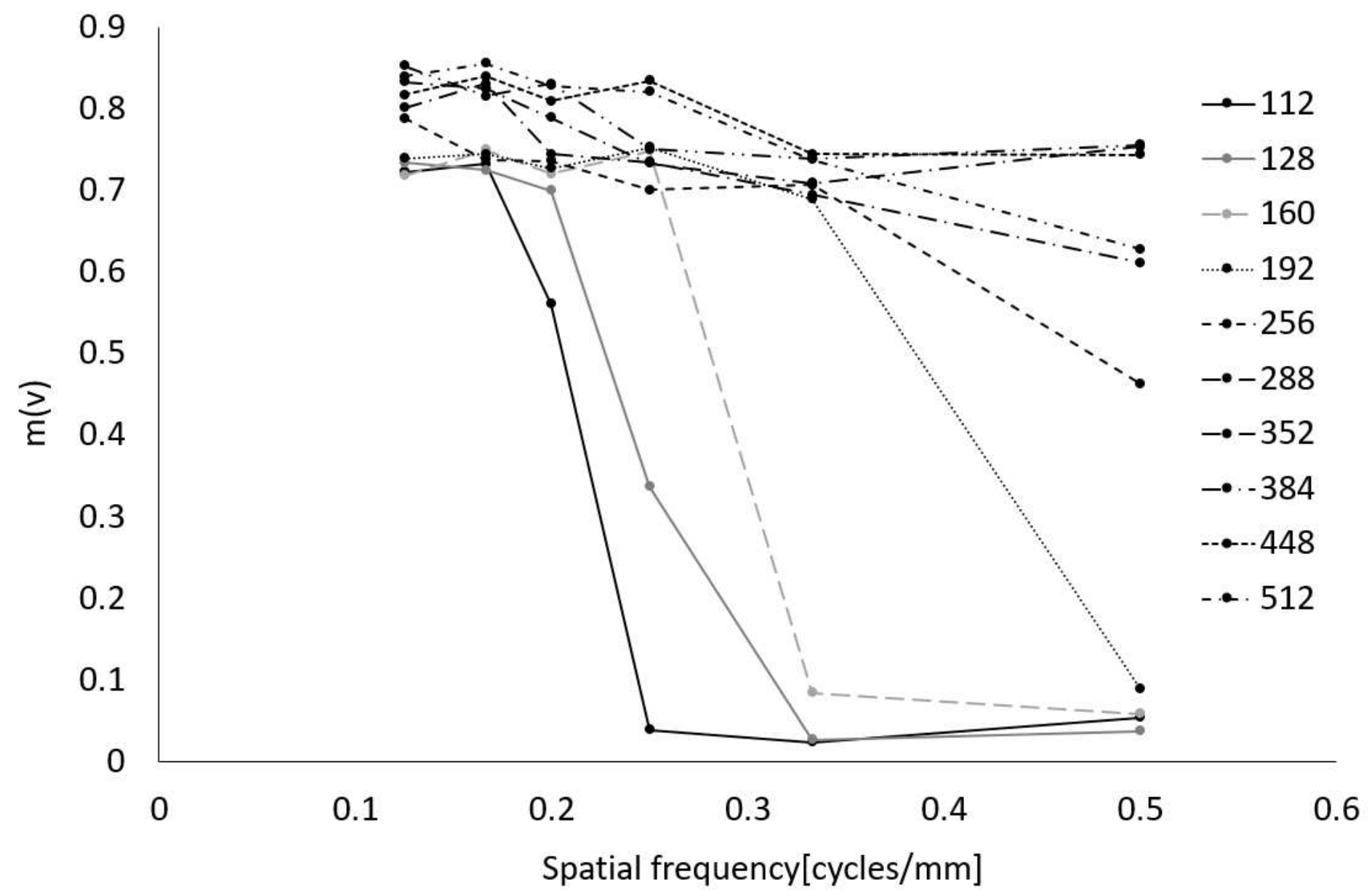

Figure 7

Relationship between spatial frequency and evaluated $\mathrm{m}(\mathrm{v})$ at different resolutions. 


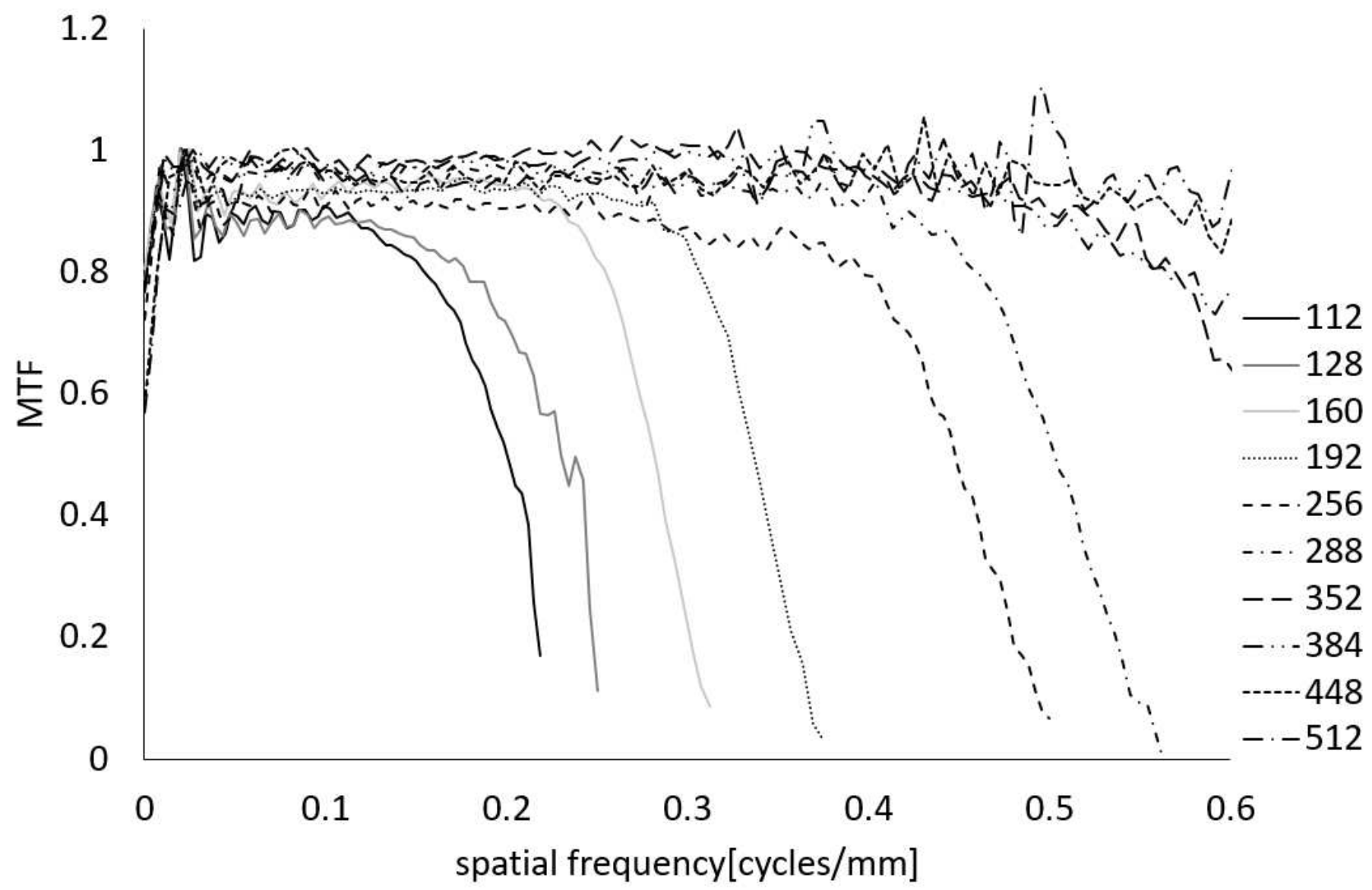

Figure 8

Relationship between spatial frequency and MTF obtained using edge method for different resolutions.
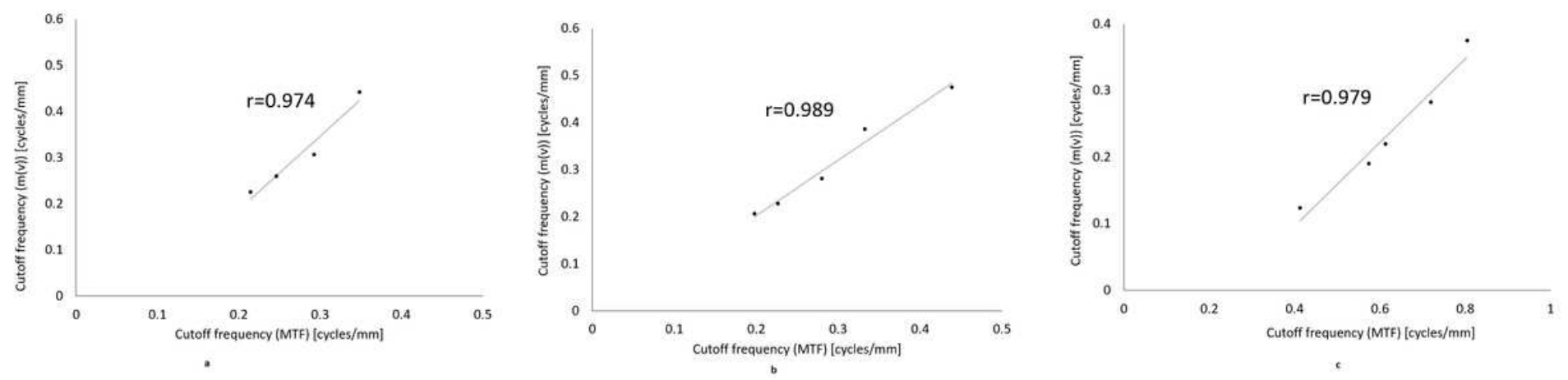

Figure 9

a. Relationship between $\mathrm{m}(\mathrm{v})$ and MTF cutoff frequency at 0.3. b. Relationship between $\mathrm{m}(\mathrm{v})$ and MTF cutoff frequency at 0.5 . c. Relationship between $\mathrm{m}(\mathrm{v})$ and MTF cutoff frequency at 0.8 .

\section{Supplementary Files}

This is a list of supplementary files associated with this preprint. Click to download. 
- Table.pdf 